\title{
Focused Laser Spike Dewetting as a Rheology Method for Soft Matter Thin Films
}

Tianxing Ma, Alex Liu, Michael Nitzsche, Kyle Buznitsky, Adithya Sridhar, Dennis Chacko, and Jonathan Singer Mechanical and Aerospace Engineering, Rutgers, the State University of New Jersey, Piscataway, New Jersey 08854, USA

\author{
Yi Jin and Zahra Fakhraai \\ Department of Chemistry, University of Pennsylvania, Philadelphia, Pennsylvania 19104, USA
}

(Dated: April 11, 2021)

\begin{abstract}
Focused laser spike (FLaSk) dewetting employs a localized heat source to create thermocapillaryinduced trench-ridge morphologies. By using a universal heating substrate to create a materialindependent thermal profile coupled with optical microscopy, we have studied the dewetted ridge feature for several distinct glassy thin films. The evolution of the ridge's radius over time can be modeled using stretched exponential functions to derive a maximum dewetted radius and a characteristic decay time. The characteristic decay time shows a super-Arrhenius behavior resembling viscosity change during the glass transition process. An effective viscosity is defined by balancing the thermocapillary Marangoni stress using the mean temperature in the melt pool, indicating clear signature of composition. In this way, we have demonstrated that FLaSk dewetting as a rheology method can be employed for high-throughput analysis of glassy thin film materials at high temperature and shear.
\end{abstract}

\section{INTRODUCTION}

The rheological properties of soft matter thin films are vital for their effective processing, being equally critical in lab scale preparation techniques like spin coating as in industrial scale techniques such as blade coating, and gravure printing. At the same time, nanoscale thin films supported on substrates typically exhibit different properties from their bulk form, due to the enhanced mobility of the free surface and altered film/substrate interactions. As new functional soft material thin films emerge, it is necessary to develop techniques to analyze the rheology properties of these materials at high throughput. Additionally, it is also of interest to investigate thin films systems with complex behaviors, such as nanoparticle composites, liquid crystals, and phase-separating blends. Similarly, additive manufacturing with high-performance bulk metallic glasses (BMGs) requires rapid extrusion before the BMGs crystallize and become immobile. 1] That shear accelerates BMG crystallization, 2 making this process more difficult to execute. Thorough exploration of the processing parameter space for such materials will unlock new opportunities in the biomedical, aerospace, and microelectronics sectors.

Here, we propose the measurement of thermocapillary (TC) shear as a materials-agnostic mechanical activation method to probe thin film materials. Previous research introduced focused laser spike (FLaSk) heating to generate thermal gradients up to $\sim 1000 \mathrm{~K} / \mathrm{\mu m}$, corresponding to $10 \mathrm{MPa}$ of surface shear, for studying the dewetting of polymeric $3-7$, metallic 8,9 and semiconductor thin films 10, 11, and the arrangement of block copolymers 12, 13]. In the FLaSk heating process, continuous wave laser excitation produces TC gradients with

\footnotetext{
* jonathan.singer@rutgers.edu
}

a high degree of spatial and temporal control. Through local softening, samples that are solid both before and after excitation lock in the effects of the exposure, such as dewetting or annealing. This is the key advantage of the approach: thermal gradients simultaneously provide the mobility and driving force for assembly. Further, FLaSk allows for the evaluation of thin film properties in almost inaccessible (high temperature, high shear) conditions. Current methods for studying thin films cannot access this regime and require lengthy evaluations of materials on a whole-film level. 14 20] As FLaSk experiments can occur across microns, a single microscope image can potentially measure hundreds of experimental conditions simultaneously, facilitating high-throughput analysis. This potential has been partially demonstrated by our recent work, where laser spike annealing is employed to control cooling rates spanning orders of magnitude to rapidly probe the optimal glass formability on a metallic glass compositional library containing 200 combinations of alloys on a 4-inch wafer. 21.

In this letter, we demonstrate using FLaSk TC dewetting for characterization of the rheological behavior of different polymer films. Films with different materials are spin coated onto a universal heating substrate (UHS) that establishes a material-independent thermal field. The FLaSk excitation controls the material temperature and simultaneously provides a thermal gradient field $\nabla T$, which further induces a time-independent TC stress $\beta \nabla T$ on the free surface of the film. By analyzing the evolution of the radius of the dewetted features over time at different heating conditions under the TC stress, we can extract the decay time, activation energy, and effective viscosity of TC dewetting; we hereby demonstrate examples of several materials with different molecular structures: polystyrene (PS) above and below its entanglement limit, molecular glass $N, N^{\prime}$-bis $(3-$ methylphenyl)- $N, N^{\prime}$-diphenylbenzidine (TPD), and SU8 (Hexion EPON Resin) under decades of shear rate at 
different temperatures. Thereby, we demonstrate capability of FLaSk thermocapillary dewetting as a fast localized rheology tool for the evaluation of shear stress in thin films.

\section{METHODS}

\section{A. Experimental}

\section{Fabrication of the universal heating substrates}

$600 \mu \mathrm{m}$ thick sapphire substrates (University Wafer 2561) were cleaned by acetone, isopropyl alcohol (IPA), and DI water in sequence. The substrates were then transferred to an electron beam evaporator and evaporated with $100 \mathrm{~nm}$ molybdenum and 5-10 nm aluminum in sequence. The substrates were then kept in air at ambient temperature for the oxidation of the Al layer over months to form a barrier layer. Without further inspection, a $<4 \mathrm{~nm}$ native oxide layer is assumed to form on top of the Al layer 22, 23. Using a Thorlabs power meter, $92 \%$ of laser energy was measured to be absorbed by the heating substrate.

\section{Thin film preparation}

The UHS was first cleaned with acetone, IPA, and DI water. After the cleaning, plasma cleaning at $100 \mathrm{mTorr}$ for 10 min was carried out by a plasma cleaner using air plasma. Dilute solutions of PS were prepared by dissolving in propylene glycol methyl ether acetate. PS thin films with four different molecular weights (MWs) were spin coated onto the fresh-cleaned UHSs. The same cleaning and spin coating procedures were repeated on Si wafers. TPD thin films and SU-8 resin thin films were made using the same method with their dilute solutions in 1,2-dichlorobenzene and cyclopentanone respectively. The TPD film was annealed at $348 \mathrm{~K}$ for $15 \mathrm{~min}$ on a hot plate while the SU-8 film was annealed at 368 $\mathrm{K}$ for $1 \mathrm{~min}$. The thicknesses of the thin films on the UHS were approximated by the measured thickness of the Si substrate-supported duplicates with a Filmetrics F-40 EXR spectroscopic reflectometer (which is more accurate on Si substrates than on UHSs). The material, MW, and thickness of the films are summarized in TAB. [1.

\section{FLaSk dewetting}

The sample was placed on a three-axis $532 \mathrm{~nm}$ continuous wave (CW) Nd:YAG laser direct write system used in previous work 9 . The beam profile was manually adjusted to be in the $\mathrm{TEM}_{00}$ mode. The laser beam was focused onto the Mo layer of the UHS through a 1-mm
TABLE I. Fitting Results of Eq. 4

\begin{tabular}{ccccccccc}
\hline \hline Material & $\begin{array}{c}\mathrm{MW} \\
(\mathrm{kDa})\end{array}$ & $\begin{array}{c}h_{0} \\
(\mathrm{~nm})\end{array}$ & $\begin{array}{c}E_{A} \\
(\mathrm{~kJ} / \mathrm{mol})\end{array}$ & $\mathrm{C}$ & $R^{2}$ & $\mathrm{RMSE}$ & $\begin{array}{c}\text { Bulk } T_{g} \\
(\mathrm{~K})\end{array}$ \\
\hline PS & 4 & 150 & 111.58 & -25.98 & 0.98 & 0.32 & $346[24]$ \\
PS & 35 & 154 & 140.44 & -29.86 & 0.99 & 0.20 & $370[24]$ \\
PS & 280 & 150 & 94.98 & -15.70 & 0.96 & 0.42 & $373[25]$ \\
PS & 2000 & 154 & 100.93 & -15.07 & 0.97 & 1.35 & $374[25]$ \\
TPD & 0.52 & 150 & 224.11 & -44.46 & 0.96 & 0.64 & $330[26$ \\
SU-8 & 1.40 & 143 & 124.40 & -28.55 & 0.99 & 0.35 & $333[27$ \\
PS & 35 & 50 & 141.42 & -30.29 & 0.96 & 1.74 & $370[24]$ \\
PS & 35 & 200 & -126.38 & 16.56 & 0.74 & 0.22 & $370[24]$ \\
\hline \hline
\end{tabular}

thick borosilicate glass slide from an objective lens with 0.25 numerical aperture (NA). Before tests on each film, the focus of the beam was manually adjusted by finding the sharpest, brightest photoluminescence spot in the camera. The tilt of the sample is compensated for in software from 3 alignment points by piezoelectric $z$-stage's movement during translation. Using a customized MATLAB program, FLaSk dewetting was conducted on the UHSs covered with different thicknesses of material using laser power ranging from $20 \mathrm{~mW}$ to $210 \mathrm{~mW}$ and exposure time from $0.1 \mathrm{~ms}$ to $100 \mathrm{~s}$. The center-to-center distance between each test was at least $50 \mu \mathrm{m}$ to prevent annealing of nearby testing spots. Each power-exposure time combination was repeated at least three times on the same film. The detailed FLaSk parameters can be found in the Supporting Information (SI).

\section{Feature size characterization}

An AFM scan was conducted on one FLaSk dewetted feature, an optical image was also taken on the same feature using a Leica DM2700M optical microscope. (See SI for more details.) Comparing the profile of the AFM height map (FIG $1 \mathrm{~d}$ ) and the red channel of the RGB image (FIG 1k), we found the valleys in the red intensity correlate with the center points between the ridge peaks and the valley bottom (as indicated by the red dashed lines between FIG 1p and FIG 1, d), which should be representative enough as the FWHM of the feature similar to what has been used in previous work [5].

The valley locations of the red intensity map were then used for defining the characteristic radius of the dewetted features using a customized MATLAB code. More details can be found in the SI.

\section{B. Thermal Simulation}

The simulation of the thermal profile was done using COMSOL Multiphysics. In an axisymmetric model, the laser beam was modeled as a Gaussian-shaped boundary heat source on the bottom surface of the Mo layer with a diameter given by equation $D_{0}=\frac{4 \lambda}{\pi(N A)}$, where NA 


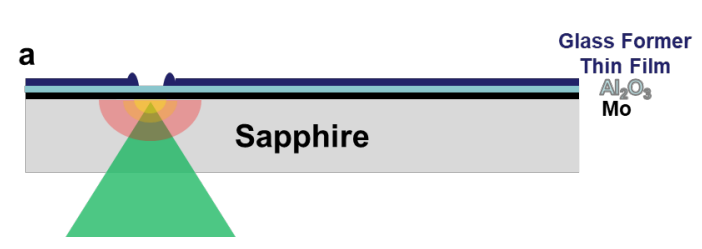

C

b
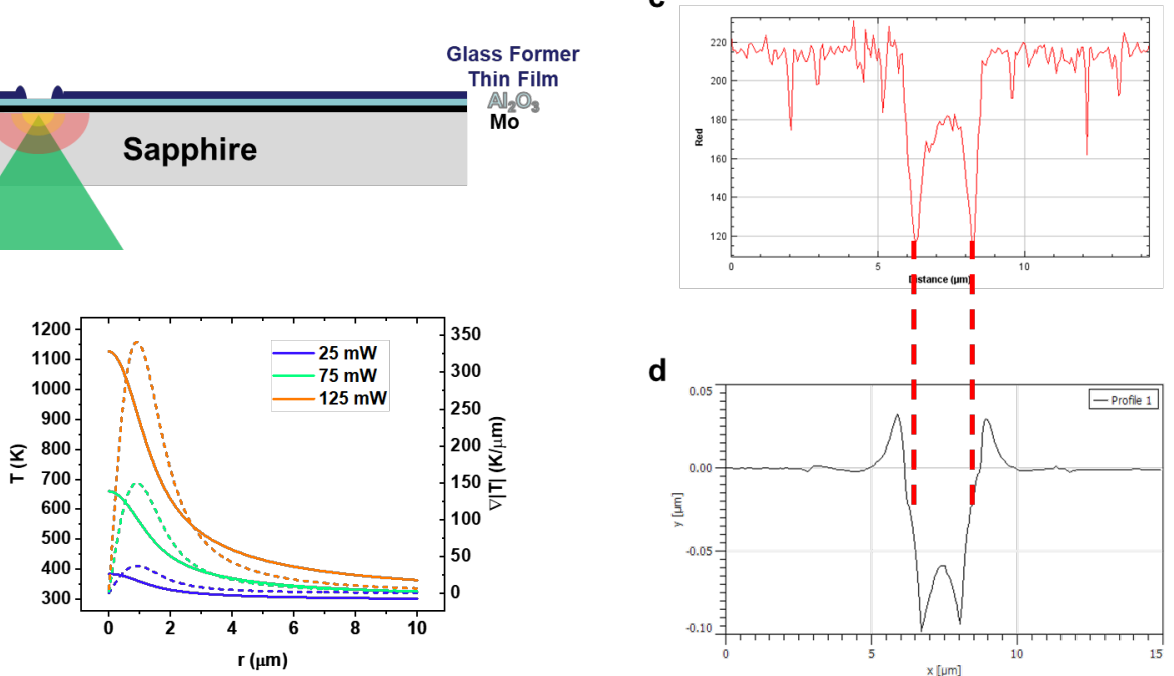

FIG. 1. (a) Schematic drawing of the experimental set up. (b) Thermal profile and thermal gradient profile on top of the UHS from three characteristic laser powers with the solid lines showing the radial temperature while the dashed lines representing the radial thermal gradient. (c) intensity profile of the red channel of the optical image of a FLaSk dewetted feature. The red dashed lines are a guidance for eyes showing the correlation between the radius defined in red channel of optical intensity map and the radius defined in the actual feature's profile. (d) AFM measured profile of the same feature of (c). (c) and (d) are measured on a dewetted feature on a $154 \mathrm{~nm}$ thick PS 2 MDa film using $170 \mathrm{~mW}$ and $1 \mathrm{~s}$ as the FLaSk parameters. The optical image and the AFM image can be found in the SI.

$=0.25$ is the numerical aperture of the objective lens and $\lambda$ is the wavelength of the laser 28]. The UHS was modeled as a three-layer structure of which the bottom is a $600 \mu \mathrm{m}$ thick sapphire, the center layer is a $100 \mathrm{~nm}$ thick Mo layer and the capping layer is set as $10 \mathrm{~nm}$ thick of sapphire. The simulation cell's radius was set to 350 um. A constant temperature boundary condition of $T=$ $T_{a m b}=293.2 \mathrm{~K}$ was set to the bottom of the substrate while a natural convection boundary condition was set to the side edges and the top surface of the substrate.

The FEM thermal simulation gives a linear relation between the laser power and the max temperature the UHS has reached during the FLaSk process:

$$
T_{\max }=6.673 \mathrm{~K} / \mathrm{mW} \cdot P+293.2 \mathrm{~K}
$$

where the $T_{\max }$ is the $\max$ temperature and $P$ is the power. More details of the calibration process can be found in the SI.

To calibrate the laser power-max temperature relation, laser heating experiments with $\geq 1$ s exposure time and power up to $500 \mathrm{~mW}$ power were conducted on different crystalline materials deposited on UHS (see SI for more details). The samples were then examined by optical microscopy and/or AFM scans to find laser induced melting at different powers to correlate their melting point with the first experimental sign of melting.

\section{RESULTS AND DISCUSSION}

\section{A. Radius Evolution}

FIG. 2 shows the dewetted radius as a function of exposure time for the tested thin films. At a constant laser power (or equivalently, a constant $T_{\max }$ ), the dewetted radii grow rapidly during the initial stage. A radius then asymptotes at a maximum radius for each power. It is worth noting that, while it only takes less than $20 \mu \mathrm{s}$ for the $T_{\max }$ to reach its steady-state value, the spatial extent of dewetting due to the sharp gradient covers a considerable range of temperatures (see SI for more details). This means that a significant portion of the evolution occurs at a lower material temperature. Thus a more representative temperature is necessary for the characterization of dewetted feature evolution.

To capture these growth features of the radius, we employed a stretched exponential fit or the KohlrauschWilliams-Watts (KWW) function which is typically adopted for the description of relaxation processes in disordered systems 29 32, for example, the glass transition process of polymer 31,32 .

$$
R=R_{\max }\left(1-\exp \left(-\left(\frac{t}{\tau}\right)^{\alpha}\right)\right)
$$

where $R$ is the dewetted radius identified by the image processing, $R_{\max }$ is the asymptotic maximum radius of dewetting over infinite time, $\tau$ is the characteristic decay 

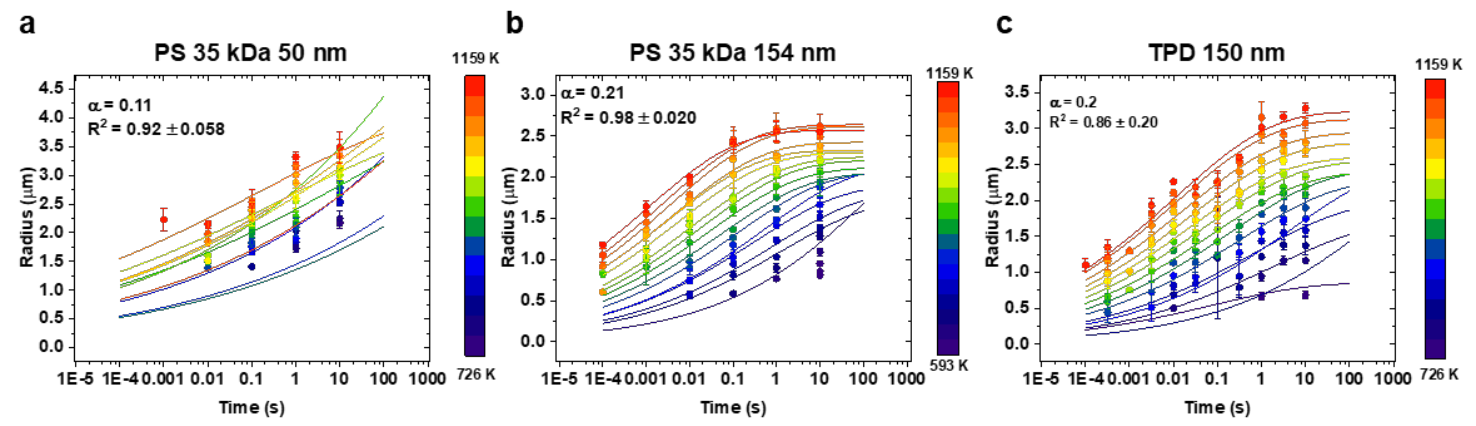

FIG. 2. Radii evolution of three characteristic films. Each color represents a laser power or $T_{\max }$. The dots are showing the experimental results at different time steps. The solid lines are the fitted KWW models using Equation 2 . The $\alpha$ and $R^{2}$ are shown in the insets. (a) $50 \mathrm{~nm}$ PS $35 \mathrm{kDa}$ film (b) $154 \mathrm{~nm}$ PS $35 \mathrm{kDa}$ film (c) $150 \mathrm{~nm}$ TPD film. Results of other films can be found in the SI

time constant, and the stretching exponent $\alpha$ is an empirical fitting parameter, optimized by the $R^{2}$ value of the fit.

Based on the max radius $R_{\max }$, we can further define a mean temperature $T_{\text {mean }}$ as the temperature metric for characterization of feature evolution:

$$
T_{\text {mean }}=\frac{1}{\pi\left(R_{\max }\right)^{2}} 2 \pi \int_{0}^{R_{\max }} T(P, R, t) R d R
$$

As seen in FIG 1p, for a $R_{\max }$ that is between $1 \mu \mathrm{m}$ and $5 \mu \mathrm{m}$ (where most $R_{\max } \mathrm{s}$ lie in for this work), the magnitude change of the thermal gradient is within one decade for most laser powers. Thus, the use of a constant mean temperature during the whole dewetting process should suffice for characterization of the feature evolution under a fixed stress field.

The decay time $\tau$ is plotted against $T_{\text {mean }}$, in FIG 3 . It can be seen that $\ln \tau$ follows a linear relation with $1 / T_{\text {mean }}$. Within the same temperature range, the $\tau$ of $\sim 150 \mathrm{~nm}$ PS films follows the MW order with higher MW having a longer $\tau$ (FIG. 3a). For PS $35 \mathrm{kDa}$ films with different thickness(FIG. 3p), the thickest film (200 $\mathrm{nm}$ ) failed to get data at temperatures spanning a wide range - most points cluster at a small range of $T_{\text {mean }}$. In addition, they are lying several decades higher than the extrapolated line of the thinner film $(50 \mathrm{~nm}$ and $150 \mathrm{~nm})$ models. At this thickness, it is possible that the temperature may not be uniform through the thickness at the early stage of the dewetting. The $\tau$ of the thinner films (50 $\mathrm{nm}$ and $150 \mathrm{~nm}$ ), on the other hand, collapse onto a single line with the thinnest $(50 \mathrm{~nm})$ films extending the trend to lower $T_{\text {mean }}$. The unentangled PS (4 kDa) and the SU-8 films display the same behavior as the PS $35 \mathrm{kDa}$ films with $50 \mathrm{~nm}$ and $150 \mathrm{~nm}$ thicknesses. Although the TPD has a low molecular weight, it possesses a longer decay time than the SU-8 and low MW PS film. The slope of the TPD film is also higher than both the SU-8 and PS films. This might come from its molecular structure being different from PS and SU-8 since the difference between the structures of backbone and side chain can modify the relaxation process to a great extent. [33] An example can be seen from past work on the fragility and apparent activation energy of rearrangement (measured by dewetting experiments) of molecules with different structures. 34] Despite that the TPD has a similar dewetting activation energy as PS with a MW of $45.7 \mathrm{kDa}$ in bulk films [16, 35], the TPD film has a much higher FLaSk activation than the PS films as seen in TAB!

This abnormally high activation energy might be attributed to the enhanced surface mobility of TPD as the surface molecules are orientated more parallel to the free surface due to the molecular structure of TPD as compared with unentangled or slightly entangled linear polymer. 36 Thus, we speculate this more stable near surface conformation could be a source of this higher activation energy. For further characterization, the data is fit to the Adam-Gibbs relation [37:

$$
\ln \tau=\frac{E_{A}}{R T_{\text {mean }}}+C
$$

where the $E_{A}$ is the apparent activation energy and $C$ is a fitting constant. $R$ is the universal gas constant. This super-Arrhenius relation resembles the VogelFulcher-Tammann equation that describes the relaxation of glass formers 38 40]:

$$
\ln \tau=\frac{T_{A}}{T-T_{0}}+\ln \tau_{\infty}
$$

where $T_{0}$ is the Vogel temperature which typically takes the value of the Kauzmann temperature. The $T_{A}$ is an activation temperature that scales with $E_{A} / k_{B} . k_{B}$ is the Boltzmann constant while $\tau_{\infty}$ is the high temperature limit of the decay time.

Comparing Equations 4 and 5 , we expect the fitting parameter $C$ to play a same role as $\tau_{\infty}$ while $E_{A} / R$ having the same role as $T_{A}$. The fitting result using equation 4 has been summarized in TABLE []

As seen in TABLE If with the $R^{2}$ values generally larger than 0.95 (except for the PS $35 \mathrm{kDa} 200 \mathrm{~nm}$ film), 

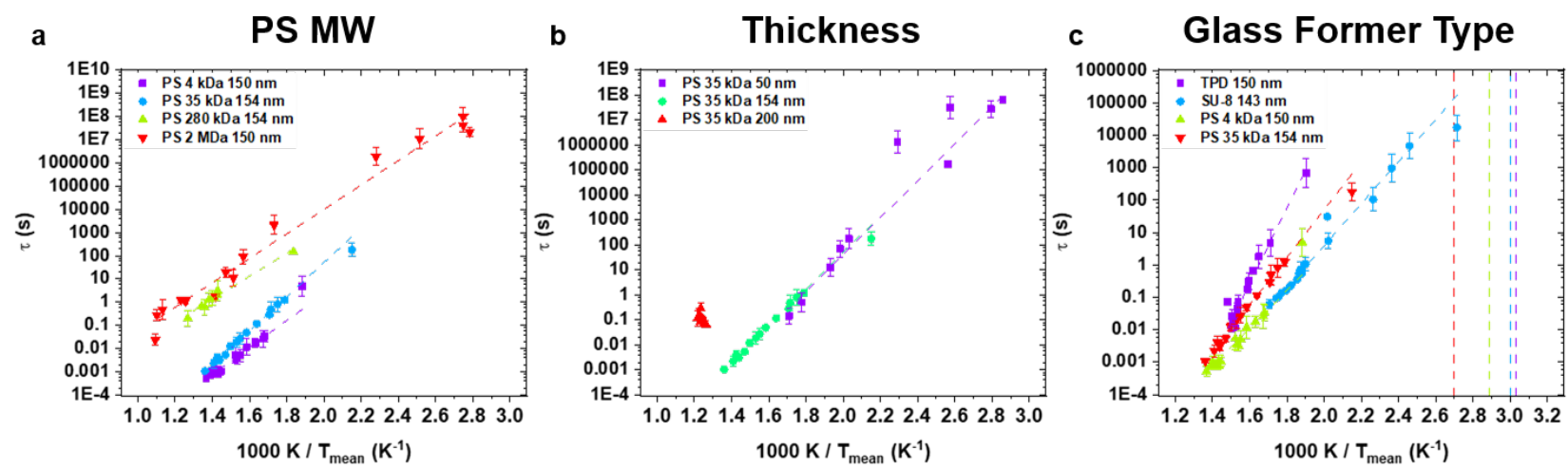

FIG. 3. Decay time versus mean temperature:(a) Comparison between PS of different MW. (b) Comparison between PS 35 $\mathrm{kDa}$ film with different film thickness (c) Comparison between different glass formers. The vertical dashed lines are showing the glass transition temperature of corresponding bulk materials.

equation 4 can sufficiently describe the TC dewetting behavior of thin films in the broad temperature range of these experiments $(350-908 \mathrm{~K})$.

Further, this indicates that the $T_{\text {mean }}$ is capable of characterizing dewetting over a sizable range of $R_{\max }$ values. Both of these statements even hold with the unexpectedly high values of TPD, indicating that the observed result is a robust behavior.

\section{B. Effective viscosity of thermocapillary dewetting}

The lubrication equation is given as follows:

$$
\frac{d h}{d t}=-\nabla \cdot\left(\frac{h^{2} \beta \nabla T}{2 \mu(T)}+\frac{h^{3}}{3 \mu(T)} \nabla\left(\gamma \nabla^{2} h+\frac{d V}{d h}\right)\right)
$$

If we neglect the second and third terms on the right hand side of Equation 6, and combine it with the continuity equation

$$
\frac{1}{r} \frac{\partial}{\partial r}\left(r \rho \frac{d r}{d t}\right)+\frac{\partial}{\partial z}\left(\rho \frac{d h}{d t}\right)=0
$$

we can get an effective viscosity $\mu$ of the thermocapillary dewetting:

$$
\mu(T) \sim-\tau \frac{\beta \nabla T}{2} \cdot \frac{h}{r}
$$

The validity of neglecting the second and third terms of the equation 6 is discussed in the SI. The $\beta$ term in equation 6 and equation 8 is the thermal Marangoni coefficient. Using $\beta$ from literature [16, 41, the effective viscosity can be seen in FIG 4 .

As shown in FIG. 4a, within the same testing temperature range, the VFT model of the bulk materials (dashed lines) can be approximated as a linear relation between viscosity and $1 / T$ in a semi-log plot. The effective viscosity inherits the linear relation between the decay time with $1 / T_{\text {mean }}$ from Equation 8 At higher temperature, the viscosity of PS films is close to their theoretical value of bulk form. However, the slope is different. The increase of $T_{\text {mean }}$, not only provides more energy to overcome the activation energy needed for the relaxation of the glass, but also provides a larger thermocapillary Marangoni driving stress $\beta \nabla T$. Consequently, the shear rate at higher $T_{\text {mean }}$ is also higher. For example, using the datapoint at the highest $T_{\text {mean }}$ of the PS 35 $\mathrm{kDa} 150 \mathrm{~nm}$ film, at $T_{\text {mean }}=735 \mathrm{~K}$, with a $R_{\max }$ of 2.57 $\mu \mathrm{m}$, the $\tau$ is $\sim 90 \mu \mathrm{s}$. Even if we assume a linear growth of the radii when the time reaches $\tau$, we can still get a shear rate of $0.67 R_{\max } /\left(h_{0} \tau\right)=1.28 \times 10^{5} \mathrm{~s}^{-1}$. While at $T_{\text {mean }}=559 \mathrm{~K}$, the shear rate is only $6.33 \mathrm{~s}^{-1}$. As the shear rate spans about 5 decades, we attribute the difference in the slope between the zero-shear VFT model and our data to both the temperature increase and the shear thinning of the glass formers. 42, 43] The TPD film's viscosity is much higher than its model. This unexpected result might come from the shear induced ordering of the material which has not yet been well-studied to the best understanding of the authors. The strong effect may be rooted in the fact that TPD molecules are elongated and the molecules may orient along the direction of shear, resulting in a non-trivial behavior.

Although the $\sim 150 \mathrm{~nm}$ and $\sim 50 \mathrm{~nm}$ PS films have a similar model for the $\tau$ versus temperature, ( FIG $3 \mathrm{~b}$ and TABLE.T, they evince different viscosity with the thinner one having a lower viscosity, exhibiting the enhanced kinetics observed for films under nano-confinement. 44] It is known that the near surface kinetics is enhanced due to the molecular conformation [45, 46]. The initial thickness of the film is high and is typically larger than ten times the radius of gyration $R_{g}$ (see SI) for PS films (except the $2 \mathrm{MDa}$ one) and thus would typically be deemed bulky for surface profile based measurements 46 48 taken during the initial deformation upon globally annealing. During FLaSk TC dewetting, however, with material moving from the melting pool, the dewetting experiences a full range of lower thickness(FIG 1 p), where the enhanced mobility of near-surface molecules could stimulate the flow. With the thinner films having thickness closer to 


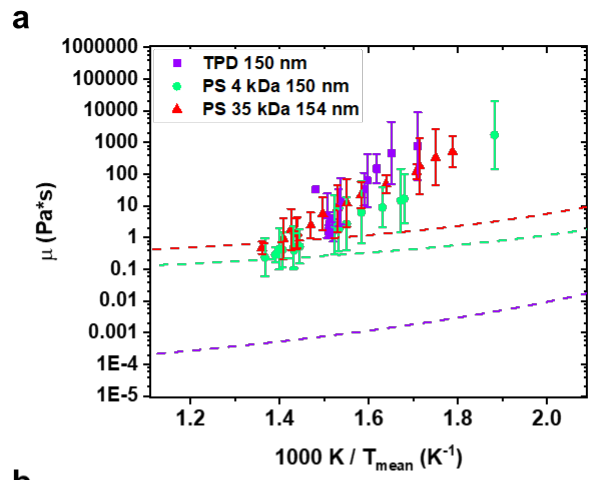

b

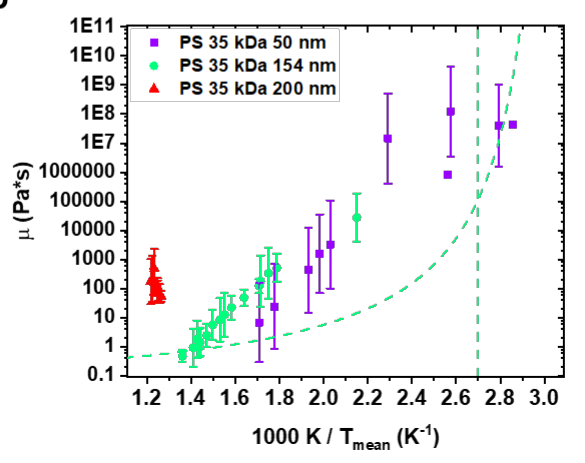

FIG. 4. Effective viscosity of thermocapillary dewetting: (a) TPD, $4 \mathrm{kDa}$ PS and $35 \mathrm{kDa}$ PS $~ 150 \mathrm{~nm}$ films. (b) PS 35 $\mathrm{kDa}$ films with different thicknesses. The vertical line shows the $T_{g}$ of bulk PS $35 \mathrm{kDa}$ films The dashed curves are the VFT models for bulk films with parameters from literature. [16]

the $R_{g}$, during the TC dewetting process, their dewetting front is more confined in the thickness direction and thus it is not surprising that the seemingly bulky films are also showing a thickness-dependent viscosity.

As demonstrated in our previous work $[9$, by combinations of different power and NA of the objective lens, the applied stress and temperature can be tuned, though a systematic study of the individual influence of stress and temperature will be left for future work.

\section{CONCLUSION}

Using dot-exposure FLaSk dewetting on a UHS combined with facile optical microscopy, a high throughput metrology method for glassy thin films under high strain rate is demonstrated. The FWHM radii of the dewetted crater generated by thermocapillary dewetting when fitted using a stretched exponential model reveals a characteristic decay time of relaxation $\tau$ of the glassy films under a constant imposed stress field. The relaxation shows a super-Arrhenius relation with mean temperature. The extracted activation energies are independent of thickness and show direct correlation with molecular weight and entanglement. Further, an effective viscosity of thermocapillary dewetting may be defined that correlates with previous theoretical and experimental results. The extracted values appear to also capture additional ordering mechanisms, as indicated by the abnormally high activation energy and resultant FLaSk viscosity of the molecular glass TPD. While the source is not immediately clear, this suggests that FLaSk rheology can be a useful tool to probe difficult to investigate regimes of thin film fluid mechanics that can be developed through more systematic study.

\section{ACKNOWLEDGMENTS}

The authors acknowledge the National Science Foundation Division Of Materials Research for partially supporting this work through award 1628407 .

\section{CITATIONS}

[1] M. A. Gibson, N. M. Mykulowycz, J. Shim, R. Fontana, P. Schmitt, A. Roberts, J. Ketkaew, L. Shao, W. Chen, P. Bordeenithikasem, J. S. Myerberg, R. Fulop, M. D. Verminski, E. M. Sachs, Y.-M. Chiang, C. A. Schuh, A. John Hart, and J. Schroers, Materials Today 21, 697 (2018)

[2] Z. Shao, J. P. Singer, Y. Liu, Z. Liu, H. Li, M. Gopinadhan, C. S. O'Hern, J. Schroers, and C. O. Osuji, Physical Review E 91 (2015), 10.1103/physreve.91.020301.

[3] O. Lyutakov, J. Tůma, I. Huttel, V. Prajzler, J. Siegel, and V. Švorčík, Applied Physics B 110, 539 (2013).

[4] R. Elashnikov, P. Fitl, V. Svorcik, and O. Lyutakov, Applied Surface Science 394, 562 (2017)

[5] J. P. Singer, S. E. Kooi, and E. L. Thomas, Journal of Polymer Science Part B: Polymer Physics 54, 225 (2016)
[6] J. P. Singer, P.-T. Lin, S. E. Kooi, L. C. Kimerling, J. Michel, and E. L. Thomas, Advanced Materials 25, 6100 (2013).

[7] A. R. Gamboa, M. P. Nitzsche, V. Saro-Cortes, T. Ma, L. Lei, and J. P. Singer, MRS Advances , 1 (2018).

[8] T. Ma, J. Guzman-Pichardo, L. C. Klein, A. Jitianu, and J. P. Singer, Focused laser spike (FLaSk) thermocapillary patterning of micro/nanostructures, SPIE LASE, Vol. 10905 (SPIE, 2019).

[9] T. Ma, M. P. Nitzsche, A. R. Gamboa, V. Saro-Cortes, and J. P. Singer, ACS Applied Nano Materials 2, 586 (2019)

[10] T. Schwarz-Selinger, D. G. Cahill, S. C. Chen, S. J. Moon, and C. P. Grigoropoulos, Physical Review B 64, 155323 (2001), pRB. 
[11] J.-H. Yoo, J. B. In, C. Zheng, I. Sakellari, R. N. Raman, M. J. Matthews, S. Elhadj, and C. P. Grigoropoulos, Nanotechnology 26, 165303 (2015).

[12] J. P. Singer, K. W. Gotrik, J.-H. Lee, S. E. Kooi, C. A. Ross, and E. L. Thomas, Polymer 55, 1875 (2014)

[13] P. W. Majewski and K. G. Yager, ACS Nano 9, 3896 (2015)

[14] H. Wang, J. L. Hor, Y. Zhang, T. Liu, D. Lee, and Z. Fakhraai, ACS Nano 12, 5580 (2018).

[15] J. L. Hor, H. Wang, Z. Fakhraai, and D. Lee, Soft Matter 14, 2438 (2018).

[16] Y. Zhang, E. C. Glor, M. Li, T. Liu, K. Wahid, W. Zhang, R. A. Riggleman, and Z. Fakhraai, 145, 114502 (2016)

[17] M. Ilton, T. Salez, P. D. Fowler, M. Rivetti, M. Aly, M. Benzaquen, J. D. McGraw, E. Raphaël, K. DalnokiVeress, and O. Bäumchen, Nature Communications 9 (2018), 10.1038/s41467-018-03610-4.

[18] Y. Chai, T. Salez, J. D. McGraw, M. Benzaquen, K. Dalnoki-Veress, E. Raphael, and J. A. Forrest, Science 343, 994 (2014).

[19] J. D. McGraw, T. Salez, O. Bäumchen, Raphaël, and K. Dalnoki-Veress, Soft Matter 9, 8297 (2013).

[20] J. D. McGraw, T. Salez, O. Bäumchen, E. Raphaël, and K. Dalnoki-Veress, Physical Review Letters 109 (2012), 10.1103/physrevlett.109.128303

[21] N. Liu, T. Ma, C. Liao, G. Liu, R. M. O. Mota, J. Liu, S. Sohn, S. Kube, S. Zhao, J. P. Singer, and J. Schroers, Scientific Reports 11 (2021), 10.1038/s41598-021-83384$\mathrm{W}$.

[22] K. R. Lawless, Reports on Progress in Physics 37, 231 (1974)

[23] P. Dumas, J. P. Dubarry-Barbe, D. Rivière, Y. Levy, and J. Corset, 44, C10 (1983).

[24] A. A. Miller, 6, 1161 (1968)].

[25] C. J. Ellison, M. K. Mundra, and J. M. Torkelson, 38, 1767 (2005)

[26] Y. Zhang and Z. Fakhraai, Proceedings of the National Academy of Sciences , 201701400 (2017)

[27] S. Kim, S. A. Hewlett, C. B. Roth, and J. M. Torkelson, 30, $83(2009)$
[28] B. E. Saleh, M. C. Teich, and B. E. Saleh, Fundamentals of photonics, Vol. 22 (Wiley New York, 1991).

[29] R. Kohlrausch, 167, 56 (1854).

[30] G. Williams and D. C. Watts, Transactions of the Faraday Society 66, 80 (1970).

[31] G. B. McKenna and S. L. Simon, Macromolecules 50, 6333 (2017)

[32] S. L. Simon, J. W. Sobieski, and D. J. Plazek, Polymer 42, 2555 (2001)

[33] A. P. Sokolov, V. N. Novikov, and Y. Ding, Journal of Physics: Condensed Matter 19, 205116 (2007).

[34] K. Kunal, C. G. Robertson, S. Pawlus, S. F. Hahn, and A. P. Sokolov, Macromolecules 41, 7232 (2008).

[35] E. C. Glor, R. J. Composto, and Z. Fakhraai, Macromolecules 48, $6682(2015)$

[36] S. S. Dalal, D. M. Walters, I. Lyubimov, J. J. De Pablo, and M. D. Ediger, Proceedings of the National Academy of Sciences 112, 4227 (2015)

[37] G. Adam and J. H. Gibbs, The Journal of Chemical Physics 43, 139 (1965).

38 H. Vogel, Phys. Z. 22, 645 (1921).

[39] G. Tammann and W. Hesse, Zeitschrift für anorganische und allgemeine Chemie 156, 245 (1926).

[40] G. S. Fulcher, Journal of the American Ceramic Society 8, 339 (1925)

[41] Z. Pu, Oxford University Press, Inc , 832 (1999).

[42] R. L. Ballman and R. H. M. Simon, 2, 3557 (1964).

[43] W.-S. Guan and H.-X. Huang, Polymer Engineering Science 53, 1563 (2013)

[44] S. Napolitano, E. Glynos, and N. B. Tito, Reports on Progress in Physics 80, 036602 (2017).

[45] O. K. C. Tsui and H. F. Zhang, Macromolecules 34, 9139 (2001).

[46] Z. Yang, A. Clough, C.-H. Lam, and O. K. C. Tsui, Macromolecules 44, 8294 (2011)

[47] Z. Yang, Y. Fujii, F. K. Lee, C. H. Lam, and O. K. C. Tsui, Science 328, 1676 (2010).

[48] F. Chen, D. Peng, C.-H. Lam, and O. K. C. Tsui, Macromolecules 48, 5034 (2015) 